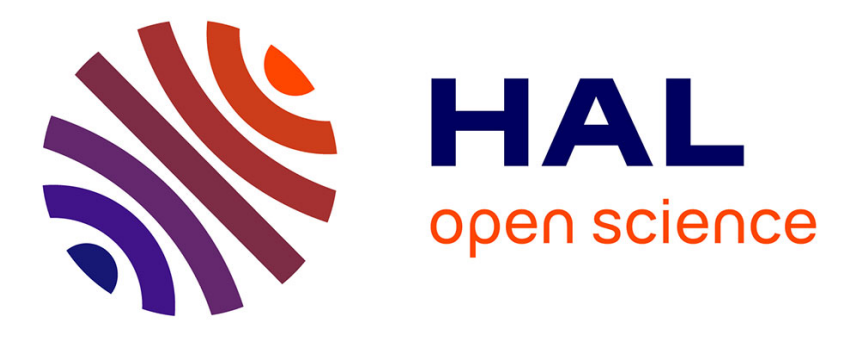

\title{
Transepithelial photorefractive intrastromal corneal crosslinking versus photorefractive keratectomy in low myopia
}

Safa El Hout, Myriam Cassagne, Thomas Sales de Gauzy, Stéphane Galiacy, François Malecaze, Pierre Fournié

\section{To cite this version:}

Safa El Hout, Myriam Cassagne, Thomas Sales de Gauzy, Stéphane Galiacy, François Malecaze, et al.. Transepithelial photorefractive intrastromal corneal crosslinking versus photorefractive keratectomy in low myopia. Journal of Cataract and Refractive Surgery, 2019, 45, pp.427 - 436. 10.1016/j.jcrs.2018.11.008 . hal-03486045

\section{HAL Id: hal-03486045 https://hal.science/hal-03486045}

Submitted on 20 Dec 2021

HAL is a multi-disciplinary open access archive for the deposit and dissemination of scientific research documents, whether they are published or not. The documents may come from teaching and research institutions in France or abroad, or from public or private research centers.
L'archive ouverte pluridisciplinaire HAL, est destinée au dépôt et à la diffusion de documents scientifiques de niveau recherche, publiés ou non, émanant des établissements d'enseignement et de recherche français ou étrangers, des laboratoires publics ou privés.

\section{(c) (1) $\$$}

Distributed under a Creative Commons Attribution - NonCommercial| 4.0 International 


\section{Running head: TRANSEPITHELIAL CROSS-LINKING IN LOW MYOPIA}

Authors:

Safa El hout, M. D, ${ }^{1,}$ Myriam Cassagne, M. D, M. Sc, ${ }^{1-2, *}$ Thomas Sales de gauzy,

FRCOph, ${ }^{1}$ Galiacy Stéphane, $\mathrm{PhD},{ }^{1-2}$ François Malecaze, M.D, PhD, ${ }^{1,2}$ Pierre Fournié, M.D, $\mathrm{PhD},{ }^{1-2}$

${ }^{1}$ Department of Ophthalmology, Purpan Hospital, Toulouse, France

${ }^{2}$ EA 4555, Paul Sabatier University, Toulouse, France

*Both S.E and M.C contributed equally to this work

${ }^{1}$ Department of Ophthalmology, Purpan Hospital, Toulouse, France

Financial Support: None

No conflicting relationship exists for any author

Correspondence:

Dr EL HOUT Safa, Department of Ophthalmology, Purpan Hospital, 1 place du Dr Baylac, Toulouse 31059, France. Tel: +33 (0)610249067; Fax: +33 (0)561777796; Email: nadinehout@hotmail.com 


\begin{abstract}
Purpose: To compare transepithelial (TE) photorefractive intrastromal corneal cross-linking (PiXL) and photorefractive keratectomy (PRK) in low myopia.
\end{abstract}

Setting: Monocentric study conducted in France (Purpan Hospital, Toulouse)

Design: Prospective, intraindividual ethics committee-approved, comparative nonrandomized study.

Methods: Myopic patients with manifest refraction spherical equivalent (MRSE) of - 1.00 to 2.50 diopters (D), and cylindrical component plano to $-0.75 \mathrm{D}$ were included. Dominant eye underwent PRK, and non-dominant eye TE-PiXL procedure with riboflavin (Paracel ${ }^{\circledR}$ Part 1 \& 2, Avedro Inc., Waltham, MS, USA), $30 \mathrm{~mW} / \mathrm{cm}^{2}$ pulsed UVA irradiation centered on pupil (Mosaic ${ }^{\mathrm{TM}}$ System,Avedro Inc., Waltham, MS, USA) for 16 minutes and 40 seconds and supplemental oxygen delivery mask. The primary outcome measure was the change in MRSE. Other outcome measures were uncorrected and corrected distance visual acuity (UDVA and CDVA), mean keratometry $(\mathrm{Km})$, and endothelial cell count (ECC) with a 6month follow-up. Adverse events were also assessed.

Results: Nineteen patients were included. At 6 months, mean MRSE decreased by $0.72 \pm$ $0.42 \mathrm{D}$ in TE-PiXL eyes and by $1.35 \pm 0.46 \mathrm{D}$ in PRK eyes $(P<.001)$. The mean change in UDVA was $-0.35 \pm 0.21$ LogMAR in TE-PiXL eyes and $-0.66 \pm 0.19$ LogMAR in PRK eyes $(P<.001)$. No complications were reported. ECC and CDVA were statistically unchanged.

Conclusion: PRK provided better visual and refractive outcomes than TE-PiXL. TE-PiXL however demonstrated the potential refractive effect of corneal cross-linking but with a limited magnitude of myopic correction to this point. 


\section{INTRODUCTION}

Corneal cross-linking (CXL), initially introduced by Theo Seiler et al in 2003, has emerged as an efficient method for the treatment of keratoconus, ${ }^{1}$ a progressive disorder characterized by thinning and steepening of the cornea, that leads to visual impairment. The aim of CXL is to create covalent bonds between amino-groups either within the collagen molecules or between proteoglycan core proteins and collagen in order to rigidify the anterior corneal stroma. ${ }^{2}$ In conventional CXL (C-CXL) described in the Dresden protocol, ${ }^{3}$ the corneal stroma is soaked after epithelial removal with a $0.1 \%$ riboflavin solution before exposure to a uniform beam of $365-\mathrm{nm}$ ultraviolet-A (UVA) radiation at $3 \mathrm{~mW} / \mathrm{cm}^{2}$ for 30 minutes $\left(5.4 \mathrm{~J} / \mathrm{cm}^{2}\right.$ dose). Many authors studied the efficiency of C-CXL and showed halting of keratoconus progression in $90 \%$ of cases. ${ }^{1,3-5}$ They also reported mean flattening of the steepest corneal curvature of up to 2 diopters (D) and a significant improvement of visual acuity (> 1 line). ${ }^{4,5}$ Over the last few years, the C-CXL technique has undergone various attempts at improvement: trans-epithelial or epithelium-on CXL (TE-CXL or epi-on CXL) has gained attention as a potential means of improving patient comfort and safety profile by leaving the barrier function of the epithelium intact and avoiding complications such as infectious keratitis or corneal healing disorders. Recently, studies have shown that topography-guided CXL (TG-CXL) procedures, aimed at specifically stiffening the cone area, decrease the maximal keratometry (Kmax) and improve the corrected distance visual acuity (CDVA) significantly compared to C-CXL. ${ }^{6-7}$

Nowadays, the refractive surgery techniques used for correction of low myopia are photorefractive keratectomy (PRK), laser in situ keratomileusis (LASIK), or small incision lenticule extraction (SMILE). However, PRK requires corneal de-epithelialization prior to excimer laser application. Similar to C-CXL, PRK involves a higher risk of complications 
due to epithelial debridement. Additionally, excimer laser (PRK and LASIK) and femtosecond laser (SMILE) refractive surgeries exert their therapeutic effects through tissue ablation, resulting in weakening of the intrastromal corneal matrix. ${ }^{8}$ Thus, a procedure preserving both the corneal epithelium and the stromal tissue could be an interesting improvement for low myopic patients.

The principle of localized corneal flattening through customized UVA irradiation gave rise to a new refractive application of CXL: trans-epithelial photorefractive intrastromal CXL (TE-PiXL). It consists of a planned intrastromal corneal remodeling by CXL in order to reduce the central mean keratometry and induce a myopic correction. Hence, we conducted a comparative study in a cohort of patients that underwent TE-PiXL in one eye and PRK in their other eye to assess the efficacy and safety of TE-PiXL to achieve a refractive correction of low myopia on healthy non-ectatic eyes.

\section{METHODS}

\section{Study design}

This prospective comparative non-randomized monocentric pilot cohort study, with paired eye control, was conducted in the department of Ophthalmology, at Purpan Hospital, in Toulouse, France. Approval was obtained from the Ethical Committee (ID-RCB: 2015A01526-43) and conducted in accordance with the Declaration of Helsinki.

\section{Patient population}


Inclusion criteria were 18 years of age and over; stable myopia with manifest refraction spherical equivalent (MRSE) of -1.00 to $-2.50 \mathrm{D}$, cylindrical component plano to $0.75 \mathrm{D}$; and minimal pachymetry above $480 \mu \mathrm{m}$. Patients signed an informed consent form prior to enrollment in the study. Patients with ocular diseases or previous ocular surgery, trauma or corneal condition were excluded. All patients presented for myopic correction and were eligible for corneal refractive surgery. Forme fruste keratoconus or keratoconus suspect eyes were excluded. The non-dominant eye of each patient underwent TE-PiXL. The controlateral dominant eye underwent PRK in the same session. Patients were informed of the possible undercorrection after TE-PiXL, resulting in a certain degree of anisometropia, well tolerated by most patients, but otherwise possibly requiring further PRK surgery.

\section{Surgical procedure}

TE-PiXL and PRK were performed in one session, in the same operating room.

\section{TE-PiXL}

After administration of topical anesthetics (tetracaine ${ }^{\circledR}$ and oxybuprocaine ${ }^{\circledR}$ (Théa, Clermont-Ferrand, France)), a specially designed oxygen delivery mask (Avedro Inc., Waltham, MS, USA) was placed above the eye to be treated. (Figure 1) This mask was connected to an oxygen wall outlet with a flowmeter and bubble humidifier. A $0.25 \%$ riboflavin solution with benzalkonium chloride in hydroxypropyl methylcellulose (Paracel ${ }^{\circledR}$ Part 1, Avedro Inc., Waltham, MS, USA) was applied at an interval of one drop every 90 seconds for a total of three minutes. From the fourth minute, another solution of $0.22 \%$ riboflavin (Paracel ${ }^{\circledR}$ Part 2, Avedro Inc., Waltham, MS, USA) was applied every 90 seconds for a total of six minutes. The supply of oxygen was opened at a flow rate of $1.5 \mathrm{~L} / \mathrm{min}$ two minutes before the end of riboflavin soaking. Excess riboflavin was flushed from the eye with balanced salt solution. The concentration of oxygen in the mask was measured with a sensor 
(Fibox 4 Oxygen Meter, PreSens Precsion Sensing Gmbh, Regensburg, Germany). An oxygen concentration of at least $95 \%$ was required to initiate UVA irradiation.

A CE marked (EU1504407) UVA delivery device (Mosaic ${ }^{\mathrm{TM}}$ System, Avedro Inc., Waltham, MS, USA) with integrated active pupil tracking technology was used (Figure 2). The pupil centration was chosen since angle kappa is known to be small in low myopia with low astigmatism. The stroma was irradiated with a 6-mm diameter spot of $365 \mathrm{~nm}$ UVA at 30 $\mathrm{mW} / \mathrm{cm}^{2}$ for 16 minutes and 40 seconds, pulsed at one-second intervals. The total dose was $15 \mathrm{~J} / \mathrm{cm}^{2}$. The UVA beam was centered on the pupil via the pupil tracking system. At the end of the procedure, the cornea was rinsed with balanced salt solution.

All eyes underwent the same protocol, independently of the preoperative myopic error. This protocol was based on theoretical modeling studies conducted by the device manufacturer.

\section{PRK}

After administration of topical anesthetics (tetracaine ${ }^{\circledR}$ and oxybuprocaine ${ }^{\circledR}$ (Théa, Clermont-Ferrand, France)), the central $8.0 \mathrm{~mm}$ corneal epithelium was removed using a soft rotating surgical brush. After centration was confirmed, excimer laser (Wavelight EX-500 Excimer Laser,Alcon Surgicals, Fort Worth, Texas, USA) photoablation was performed with a $6.5 \mathrm{~mm}$ optic zone.

\section{POSTOPERATIVE CARE}

At the end of the procedures, a topical antibiotic ofloxacine (Quinofree ${ }^{\circledR}$, Théa, Clermont-Ferrand, France) was administered. A bandage contact lens was only applied on PRK treated eye. The postoperative treatment included for both eyes a preservative free antibiotic ofloxacine (Quinofree ${ }^{\circledR}$, Théa, Clermont-Ferrand, France) 3 times daily for 7 days, 
a preservative free anti-inflammatory drop flurbiprofen $\left(\right.$ Ocufen $^{\circledR}$, Horus Pharma, Saint

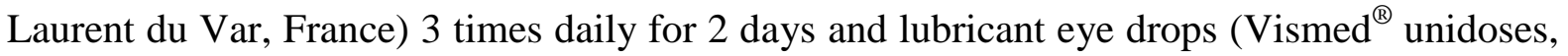
Horus Pharma, Saint Laurent du Var, France) when needed.

\section{Safety and efficacy outcomes}

The main efficacy outcome measure was the mean change in the MRSE from baseline. The secondary efficacy outcomes measures were:

- The mean change in uncorrected distance visual acuity (UDVA), assessed by a standardized scotopic decimal projection chart at a viewing distance of $5 \mathrm{~m}$ converted into logarithm of the minimum angle of resolution, $\log$ MAR notation

- The mean change in mean keratometry $(\mathrm{Km})$ on axial front curvature map of Scheimpflug tomography (Pentacam $\mathrm{HR}^{\circledR}$, Oculus, Wetzlar, Germany)

- Demarcation line depth observed by anterior segment optic coherence tomography (AS-OCT) (Spectralis ${ }^{\circledR}$, Heidelberg Engineering, Heidelberg, Germany) and measured centrally and at 4 peripheral points ( $3 \mathrm{~mm}$ temporally, nasally, in upper and lower limits), in TE-PiXL treated eye.

- The mean change in central epithelial thickness measurement on AS-OCT in both eyes.

The safety outcome measures included the best-corrected distance visual acuity (CDVA) converted into $\log$ MAR notation; the endothelial cell count (ECC) measured by specular microscopy (SP 2000P, Topcon Corporation, Tokyo, Japan), the incidence of adverse events identified by ocular examination with slit lamp biomicroscopy and the subjective patient evaluation (including pain, dryness, itching, tingling, foreign body sensation, watering, photophobia and blurred vision) performed with a visual analogic scale 
ranked from 0 to 10 for each subjective symptom. Serious side effects were defined as occurrence of infectious keratitis or more than 2 lines loss of CDVA.

\section{Patient examinations}

Patients were examined preoperatively, immediately following procedures of TEPiXL and PRK, at day 2 (D2) (to remove the bandage contact lens on PRK treated eye), day 7 (D7) to check for early complications, one (M1), three (M3) and six (M6) months postoperatively.

\section{Statistical analysis}

Comparisons of parameters with the baseline were performed using the student t-test. Data, expressed as an average \pm standard deviation (AVG \pm SD), were considered as statistically significant for a $p$ value $<0.05$. Correlation between $\mathrm{Km}$ and the demarcation line depth at 1 month was calculated using Pearson's correlation coefficient.

\section{RESULTS}

\section{Population}

Nineteen patients with a mean age of $28.2 \pm 4.8$ years were included from September 2016 through March 2017. There were no statistically significant differences between the baseline clinical characteristics of the TE-PiXL and PRK treated eyes (Table 1). 


\section{Refraction}

Table 2 and figure 3 show the MRSE changes through 6 months postoperatively with a statistically significant reduction at 6 months in TE-PiXL eyes $(\mathbf{0 . 7 2} \pm \mathbf{0 . 4 2} \boldsymbol{D}, \mathrm{p}<0.001)$, but lower than in PRK eyes $(1.35 \pm 0.46 D, p<0.001)$. Three patients had low MRSE changes after TE-PiXL, below 0.5 D.

\section{Visual acuity outcomes}

There were statistically significant $(\mathrm{p}<0.001)$ improvements in UDVA at M1, M3 and M6 postoperatively compared with baseline (Table 2 and Figure 4 A,B,C) in TE-PiXL eyes $(0.32 \pm 0,28$ decimal visual acuity $)$, but lower than in PRK eyes $(0.32 \pm 0,28$ decimal visual acuity). $69 \%$ of included patients were more satisfied with PRK treated eye. There was no regression in UDVA through follow-up visits in both techniques. 8 patients required retreatment on TE-PiXL eye after the 6-month visit.

The CDVA remained stable through 6 months postoperatively compared to the baseline in all eyes. ( $\operatorname{LogMAR}=0$, Mean decimal visual acuity=1.0).

\section{Topography outcomes}

There was a statistically significant reduction in Km from the first month, confirmed at all follow-up visits (table 2), compared to the baseline (Figure 5) in TE-PiXL eyes $(\boldsymbol{0 . 7 4} \pm$ 0.54 D, $\mathrm{p}<0.01)$ and PRK eyes $(1.15 \pm 0.53 \mathrm{D}, \mathrm{p}<0.001)$.

$18 \%$ of TE-PiXL eyes vs $73 \%$ of PRK eyes had at least 1 D decrease in mean keratometry. (Figure 6) 
The qualitative analysis of differential axial front curvature maps between the 6 months and baseline (Figure 7) showed corneal central flattening of at least $1 \mathrm{D}$, in $62.5 \%$ of TE-PiXL eyes vs $100 \%$ of PRK eyes.

\section{OCT demarcation line and epithelial thickness}

Table 3 shows the mean central and peripheral demarcation line depth at each visit. A stromal demarcation line (Figure 8) was observed in all TE-PiXL treated eyes, and was the most noticeable at M1. It subsided gradually starting from the peripheral stroma but often remained visible at M6. The correlation analysis demonstrated a statistically significant correlation between the depth of demarcation line and the $\mathrm{Km}$ reduction at $\mathrm{M} 1$ ( $\mathrm{r}=0.61$, $\mathrm{p}<0.01)$. In the same way, we noticed a correlation between the depth of demarcation line and the MRSE improvement $(\mathrm{r}=0.51, \mathrm{p}<0.05)$ (Figure 9).

There was no significant change in the mean central epithelial thickness measurements at all follow-up visits compared to the baseline in both eyes.

\section{Endothelial cell count}

There were no statistically significant differences between baseline and postoperative endothelial cell count (table 2). A blurred image of the endothelial cells (Figure 10) was obtained at M1 and M3 in TE-PiXL eyes, and then the image quality improved at M6.

\section{Side effects}


The TE-PiXL eyes were pain free postoperatively, compared with PRK eyes (100\% painful eyes at D2). A foreign body sensation (89\%) within the 2 first days postoperatively was noticed after TE-PiXL. Patients complained about moderate photophobia in both eyes (100\% of patients at D2, and $89 \%$ at D7) during the first month with a tendency to decrease over the months. There were no serious side effects. A punctate epithelial staining was noticed immediately following the TE-PiXL procedure but the epithelium was fully intact by day 2. Ulcer remained in $36.8 \%$ of PRK treated eyes at day 2.

A transient delineated corneal haze was observed in $84 \%$ of TE-PiXL treated eyes, and 5.2\% of PRK treated eyes (Figure 11). It was maximal at M1 and faded gradually. It remained slightly visible in some patients at M6.

\section{DISCUSSION}

Nowadays, corneal refractive surgery techniques are commonly used for the correction of low myopia thanks to their simplicity, efficiency and predictability. It consists of performing a customized stromal photoablation with the excimer laser. However, tissue removal impacts biomechanical properties of the cornea resulting in weakening and reduction of the stromal rigidity. ${ }^{8}$ Consequently, it is not practiced in eyes with subclinical keratoconus or thin corneas due to the risk of corneal ectasia. Several studies suggest that screening tests for refractive surgery should be done carefully, using corneal topography to exclude keratoconus suspects. ${ }^{9-10}$ However, others report that eyes with keratoconus or keratoconussuspect eyes can obtain acceptable results with refractive surgery: photorefractive keratectomy was a safe and effective procedure for keratoconus-suspect corneas over a 5year follow-up, ${ }^{11}$ topography-guided laser ablation treatment was found to improve the 
UDVA and CDVA in patients with mild to moderate degrees of keratoconus. ${ }^{12}$ But in actual practice, excimer laser keratectomy is still considered to be contraindicated in eyes with an unstable thin cornea with keratectasia. Therefore, to avoid further weakening and ectasia of the keratoconic cornea after excimer laser corneal ablation, many refractive surgeons have performed simultaneous topography-guided PRK and riboflavin-UVA CXL. ${ }^{13}$

Unlike corneal refractive surgery techniques, there is no stromal ablation in CXL. Standard CXL is known to induce a corneal flattening of approximately $2 \mathrm{D}$ in patients with keratoconus. ${ }^{3-5}$ This corneal flattening is thought to be the result of stromal stiffening induced by increased formation of covalent bonds within or between collagen molecules and proteoglycan core proteins. $^{2}$

The latest advances in the field of CXL gave rise to many variants of the C-CXL Dresden protocol, not only by modifying the protocol of riboflavin soaking through the epithelium (TE-CXL ${ }^{14}$ or iontophoresis ${ }^{15}$ ) but also by modifying the UVA irradiation protocol: reducing the time of the procedure (accelerated $\mathrm{CXL}^{16}$ ) or customizing the irradiation pattern (TG-CXL). TG-CXL has shown that greater corneal flattening can be achieved relative to C-CXL by applying cone-centered patterns of irradiation, delivered by devices allowing customized treatment plans (Mosaic KXL II® device, Avedro Inc., Waltham, MS, USA). ${ }^{6-7}$

Given the multitude of CXL protocols, finite element modeling of the cornea and the kinetics of the CXL photochemical reactions have been developed. ${ }^{17}$ These numerical models are used to simulate the post CXL topographic changes, to predict the theoretical maximum depth of the treatment and to optimize several parameters (UVA dose, irradiance, pulse interval, riboflavin concentration, and addition of supplemental oxygen). ${ }^{18}$ Seven and Dupps $^{19}$ developed and applied a biomechanical model to study potential refractive 
applications of CXL, notably the correction of astigmatism by the means of customized irradiation patterns guided by the topography.

Kanellopolous et al published the first reported case of photorefractive CXL using a high dose of UVA $\left(14 \mathrm{~J} / \mathrm{cm}^{2}\right)$ for astigmatism correction, showing a toric reduction of $0.8 \mathrm{D}$ Then, he used a peripheral irradiation profile for hyperopia correction with encouraging results. Finally, he showed a statistically significant reduction of MRSE through a feasibility study to evaluate PiXL in the treatment of low myopia. ${ }^{20}$

Elling et al ${ }^{21}$ recently published a case series evaluating PiXL for the treatment of low myopic refractive error in 24 eyes of 14 patients, using an epithelium-off (epi-off) CXL technique. While similar irradiation parameters were applied to our study $(30 \mathrm{~mW} / \mathrm{cm} 2$ pulsed UVA, dose $15 \mathrm{~J} / \mathrm{cm} 2$ ), a smaller (4mm) UVA beam diameter was used than in our study (6mm). Elling reported a MRSE reduction of $0.90 \pm 0.40 \mathrm{D}$ and a UDVA improvement of $0.34 \pm 0.20 \log$ MAR, a $0.2 \mathrm{D}$ greater reduction in mean MRSE than in our study.

Our prospective pilot study to evaluate high fluence, pupil centered TE-PiXL for the treatment of low myopia in healthy eyes was conducted using protocols developed from this modeling framework and early clinical experience. The purpose was to ascertain a proof of concept that PiXL could safely flatten the cornea and reduce myopia. The benefit of the TEPiXL technique over the epi-off PiXL technique is that in TE-PiXL, the epithelium is not removed, avoiding the risk of complications such as infectious keratitis, ${ }^{22}$ sterile infiltrates, corneal opacities and herpetic reactivation and postoperative discomfort experienced by the patient after epithelial debridement. ${ }^{23}$ In our study we have chosen, for ethical reasons, to treat only one eye per patient, the non-dominant eye, using this novel technique, limiting our sample to 19 eyes in TE-PiXL group. The dominant eye was treated using PRK. This allowed to compare TE-PiXL outcomes with those of a standard corneal refractive surgery technique. In case of under-correction with TE-PiXL, it triggers a small monovision well tolerated in 
most myopic patients. The treatment protocol for TE-PiXL used in our study induced a decrease in MRSE of $0.72 \pm 0.42 \mathrm{D}$ vs $1.35 \pm 0.46 \mathrm{D}$ in PRK eyes as well as an improvement in visual acuity of $0.35 \pm 0.21 \log$ MAR vs $0,66 \pm 0,19 \operatorname{logMAR}$ in PRK eyes. In these 2 studies, the standard deviation of the refractive outcomes suggests lesser predictability than with conventional refractive procedures, indicating that further customization of the procedure for individual patient factors, such as topography, may be required.

These results were obtained by the first month. The measurement of the central epithelial thickness preoperatively and at 1,3 and 6 months did not reveal any significant changes, indicating that the outcomes are likely due to stromal remodeling and not to epithelial variations. Furthermore, there was no significant change from the first through the sixth month, confirming that there is no early refractive regression. While some refractive methods studied in the past, such as collagen shrinkage procedures, demonstrated early regression, the findings in our study suggest the potential for refractive stability after PiXL, which must be validated with longer-term assessment.

To the best of our knowledge, our study is the first reporting TE-PiXL results with pulsed UV light and supplemental oxygen delivery. When the epithelial barrier is left intact, the epithelium slows the diffusion of both riboflavin and ambient oxygen into the cornea, thus the efficacy may be reduced compared to the standard epi-off technique. ${ }^{24}$ With the aim of maximizing the efficacy of TE-PiXL, we applied a two-part riboflavin formulation specifically marketed for use in TE-CXL procedures (ParaCel Part 1 and 2, Avedro, Inc.). This formulation contains a higher concentration of riboflavin than applied in conventional epi-off cross-linking, and contains permeability enhancing agents (associated with $0.02 \%$ benzalkonium chloride in methylcellulose) intended to loosen the epithelial junctions and aid in delivery of riboflavin to the stroma. Superficial epithelial layer could be damaged, which enhances riboflavin penetration without being completely abrasive. ${ }^{14}$ Several recent 
publications demonstrate the efficacy of this formulation in combination with accelerated cross-linking techniques to reduce maximum keratometry in patients with progressive keratoconus. Since the amount of flattening observed on average in these studies is lower than reported in some clinical studies of epi-off CXL, CXL was performed in a humidified high oxygen (>95\%) environment, using an oxygen delivery mask placed on the eye during the procedure to improve the efficiency of the epi-on procedure. The rationale for the addition of pulsed UV and supplemental oxygen is based on the understanding of CXL photochemical reactions which lead to the formation of cross-link covalent bonds within the collagen and proteoglycans molecules. ${ }^{25}$ Two types of photochemical reactions have been described. Type 1 reactions predominating under anaerobic conditions, which use excited riboflavin as substrate and generate oxygen free radicals by riboflavin photolysis, and Type 2 reactions, which occur under aerobic conditions at the beginning of the UVA irradiation and result in the formation of singlet of oxygen and subsequent photo-oxidation of stromal proteins. Rapid oxygen depletion occurs within 10 to 15 seconds of the initiation of UV in cross-linking reactions, while turning the UV light off leads to a rapid replenishment of oxygen. ${ }^{26}$ Hence, pulsing the UV light during crosslinking treatment theoretically achieves an additional oxygen concentration by slowing the rate of oxygen consumption, while the addition of supplemental oxygen at the surface increases the rate of oxygen diffusion. In silico photochemical kinetic modeling and ex vivo biomechanical analyses have previously demonstrated that the combination of pulsed irradiation of UV and an oxygen-enriched environment result in increased stromal oxygen concentration during CXL, and increased corneal stiffness. ${ }^{27}$ However, this study did not include a control group of eyes treated without oxygen, therefore we cannot conclude on oxygen effect.

A stromal demarcation line was detected on AS-OCT in all TE-PiXL eyes at M1. This line was on average deeper $(366 \pm 104 \mu \mathrm{m})$ than that described in the literature in TE-CXL 
$(105 \pm 15 \mu \mathrm{m}) .^{28}$ This may be due to both the increase in ambient oxygen concentration and higher total UVA dose. We highlighted a statistically significant correlation of the demarcation line at M1 to the mean MRSE ( $\mathrm{p}<0.05)$ and corneal flattening $(\mathrm{p}<0.01)$. The deeper this line was, the more the cornea was flattened and the greater the reduction in myopic error. Thus, this line, clearly visible at M1, seems to be an indirect indicator of CXL efficacy, as already reported in some studies. ${ }^{29}$ This correlation is supported by the very good PiXL refractive result obtained in 6 eyes which had a hyper-reflectivity of the whole thickness of the central cornea on AS-OCT. However, one may speculate that an even higher UVA dose could achieve a greater refractive effect but could have a deleterious effect on endothelium. If one believes that the observed demarcation line depth may be related to the high oxygen concentration, it may also be thought that modulation of this concentration with the same UVA total dose could affect the depth of the demarcation line. Endothelial cell counts were stable over 6 months in all eyes. However, the quality of the endothelial cells image obtained by specular microscopy was blurred until M3 in TE-PiXL eyes. This may be related to the observed stromal haze. Elling reported a decrease in cells hexagonality with an energy of $15 \mathrm{~J} / \mathrm{cm}^{2}$, he also attributes this fact to poor image quality. ${ }^{21}$ The maximum corneal and lenticular UV radiant exposure is established in ISO 15004-2 (2007), which defines light hazard protections for ophthalmic instruments. For exposure times less than $1000 \mathrm{sec}(16.7$ min), the maximum corneal and lenticular dose is $1 \mathrm{~J} / \mathrm{cm} 2$ for a Group 2 instrument. The presence of riboflavin in the cornea results in transmission of only $5 \%$ of UV light to the endothelium in an epi-off procedure. Transmission is reduced to only $3.5-4.0 \%$ in an epi-on procedure due to additional absorption in the epithelial cells themselves. Therefore, the estimated UV dose reaching the endothelial cells during a $15 \mathrm{~J} / \mathrm{cm} 2$ crosslinking treatment is $0.53-0.75 \mathrm{~J} / \mathrm{cm} 2$, depending on the presence or absence of the epithelium, which in all cases is less than the limit established in the international standard. It is also important to note that 
this standard takes into account the potential for repeated or chronic exposure to the light hazard, so the limits contain a significant additional safety buffer that is not necessarily applicable to a one-time treatment such as corneal crosslinking. Comparable studies in rabbit eyes have shown acute damage thresholds of up to $70 \mathrm{~J} / \mathrm{cm} 2$ for the lens and $42 \mathrm{~J} / \mathrm{cm} 2$ for the cornea. $^{30}$ Longer-term monitoring of these patients and of larger samples are justified to establish the safety of this procedure.

In our patients, no serious side effects were noticed. CDVA remains stable through 6 months postoperatively. The epithelium-on approach, that limits epithelial damage to superficial punctate staining immediately following the procedure, has a notable advantage since it reduces the infectious risk and greatly increases the patient's comfort. The procedure was simple for both the patient and the operator. However, the procedure lasts almost 30 minutes while other corneal refractive procedures last just a few minutes. Hence, opportunities to reduce the time of the procedure is a future improvement to be considered and evaluated.

Finally, we have continued to monitor our enrolled patients. In the case of residual refractive error after TE-PiXL, we suggested a PRK retreatment for patients wishing to achieve emmetropia. 8 patients were treated with PRK after M6, and based on the last (and lower) refraction, had, at 6 weeks post-PRK, a plano refraction and mean UDVA of 0.0 $\log$ MAR. Postoperative biomicroscopy examination did not reveal sub-epithelial haze or any other adverse effects following retreatment. 


\section{CONCLUSION}

Our study showed that high dose, pupil-centered TE-PiXL reduces low myopia. TEPiXL seems to be a minimally invasive technique for the treatment of mild refractive error, particularly as an option for patients with thin corneas (treatment-naïve or after refractive surgery) or suspicious corneal topography contraindicated for conventional refractive surgery.

However, broader cohorts with long-term data are needed to confirm the safety of the procedure and the stability of the results.

Efficiency of TE-PiXL is still limited and inferior to epi-off CXL and PRK. It could be improved in the future by modulating the various parameters thanks to the development of numerical modeling of cornea and CXL. Alternate beam patterns or integration of corneal topography data may improve treatment accuracy, particularly in the correction of astigmatism. 


\section{What is known}

- PiXL is a new approach for the correction of low myopic errors based on the flattening of the central cornea after CXL.

- Epi-off PiXL has been studied on small cohorts reporting myopia reduction and UDVA improvement. Trans epithelial PiXL, avoiding complications risk has not been widely studied yet.

\section{What this paper adds}

- Trans epithelial PiXL is effective to reduce low myopic error in healthy eyes. No complications occured during the 6-month postoperative period.

- This procedure could be in the future, a non-invasive refractive procedure for low myopia, especially on thin corneas. 


\section{BIBLIOGRAPHY}

1. Wollensak G, Spoerl E, Seiler T. Riboflavin/ultraviolet-a-induced collagen crosslinking for the treatment of keratoconus. Am J Ophthalmol. 2003;135(5):620-627.

2. Spoerl E, Huhle M, Seiler T. Induction of cross-links in corneal tissue. Exp Eye Res. 1998;66(1):97-103. doi:10.1006/exer.1997.0410

3. Wollensak G. Crosslinking treatment of progressive keratoconus: new hope. Curr Opin Ophthalmol. 2006;17(4):356-360. doi:10.1097/01.icu.0000233954.86723.25

4. Raiskup-Wolf F, Hoyer A, Spoerl E, Pillunat LE. Collagen crosslinking with riboflavin and ultraviolet-A light in keratoconus: long-term results. J Cataract Refract Surg. 2008;34(5):796-801. doi:10.1016/j.jcrs.2007.12.039

5. Caporossi A, Mazzotta C, Baiocchi S, Caporossi T. Long-term results of riboflavin ultraviolet a corneal collagen cross-linking for keratoconus in Italy: the Siena eye cross study. Am J Ophthalmol. 2010;149(4):585-593. doi:10.1016/j.ajo.2009.10.021

6. Cassagne M, Pierné K, Galiacy SD, Asfaux-Marfaing M-P, Fournié P, Malecaze F. Customized Topography-Guided Corneal Collagen Cross-linking for Keratoconus. J Refract Surg Thorofare NJ 1995. 2017;33(5):290-297. doi:10.3928/1081597X20170201-02

7. Seiler TG, Fischinger I, Koller T, Zapp D, Frueh BE, Seiler T. Customized Corneal Cross-linking: One-Year Results. Am J Ophthalmol. 2016;166:14-21. doi:10.1016/j.ajo.2016.02.029

8. Dawson DG, Randleman JB, Grossniklaus HE, et al. Corneal ectasia after excimer laser keratorefractive surgery: histopathology, ultrastructure, and pathophysiology. Ophthalmology. 2008;115(12):2181-2191.e1. doi:10.1016/j.ophtha.2008.06.008

9. Randleman JB, Caster AI, Banning CS, Stulting RD. Corneal ectasia after photorefractive keratectomy. J Cataract Refract Surg. 2006;32(8):1395-1398. doi:10.1016/j.jcrs.2006.02.07

10. Malecaze F, Coullet J, Calvas P, Fournié P, Arné J-L, Brodaty C. Corneal ectasia after photorefractive keratectomy for low myopia. Ophthalmology. 2006;113(5):742-746. doi:10.1016/j.ophtha.2005.11.023

11. Guedj M, Saad A, Audureau E, Gatinel D. Photorefractive keratectomy in patients with suspected keratoconus: five-year follow-up. J Cataract Refract Surg. 2013;39(1):66-73. doi:10.1016/j.jcrs.2012.08.058

12. Koller T, Iseli HP, Donitzky C, Ing D, Papadopoulos N, Seiler T. Topography-guided surface ablation for forme fruste keratoconus. Ophthalmology. 2006;113(12):21982202. doi:10.1016/j.ophtha.2006.06.032 
13. Shetty R, Nuijts RMMA, Nicholson M, et al. Cone location-dependent outcomes after combined topography-guided photorefractive keratectomy and collagen cross-linking. Am J Ophthalmol. 2015;159(3):419-425.e2. doi:10.1016/j.ajo.2014.11.020

14. Stojanovic A, Chen X, Jin N, et al. Safety and efficacy of epithelium-on corneal collagen cross-linking using a multifactorial approach to achieve proper stromal riboflavin saturation. J Ophthalmol. 2012;2012:498435. doi:10.1155/2012/498435

15. Bikbova G, Bikbov M. Transepithelial corneal collagen cross-linking by iontophoresis of riboflavin. Acta Ophthalmol (Copenh). 2014;92(1):e30-34. doi:10.1111/aos.12235

16. Tomita M, Mita M, Huseynova T. Accelerated versus conventional corneal collagen crosslinking. J Cataract Refract Surg. 2014;40(6):1013-1020. doi:10.1016/j.jcrs.2013.12.012

17. Roy AS, Dupps WJ. Patient-specific computational modeling of keratoconus progression and differential responses to collagen cross-linking. Invest Ophthalmol Vis Sci. 2011;52(12):9174-9187. doi:10.1167/iovs.11-7395

18. Kling S, Hafezi F. An Algorithm to Predict the Biomechanical Stiffening Effect in Corneal Cross-linking. J Refract Surg Thorofare NJ 1995. 2017;33(2):128-136. doi:10.3928/1081597X-20161206-01

19. Seven I, Roy AS, Dupps WJ, Jr. Patterned corneal collagen crosslinking for astigmatism: Computational modeling study. J Cataract Refract Surg. 2014;40(6):943. doi:10.1016/j.jcrs.2014.03.019

20. Kanellopoulos AJ. Novel myopic refractive correction with transepithelial very highfluence collagen cross-linking applied in a customized pattern: early clinical results of a feasibility study. Clin Ophthalmol Auckl NZ. 2014;8:697-702. doi:10.2147/OPTH.S59934

21. Elling M, Kersten-Gomez I, Dick HB. Photorefractive intrastromal corneal crosslinking for the treatment of myopic refractive error: Findings from 12-month interim prospective study using an epithelium-off protocol. J Cataract Refract Surg. 2018;44(4):487-495. doi:10.1016/j.jcrs.2018.01.022

22. Schallhorn JM, Schallhorn SC, Hettinger K, Hannan S. Infectious keratitis after laser vision correction: Incidence and risk factors. J Cataract Refract Surg. 2017;43(4):473479. doi:10.1016/j.jcrs.2017.01.017

23. Koller T, Mrochen M, Seiler T. Complication and failure rates after corneal crosslinking. J Cataract Refract Surg. 2009;35(8):1358-1362. doi:10.1016/j.jcrs.2009.03.035

24. Wollensak G, Iomdina E. Biomechanical and histological changes after corneal crosslinking with and without epithelial debridement. J Cataract Refract Surg. 2009;35(3):540-546. doi:10.1016/j.jcrs.2008.11.036

25. Kamaev P, Friedman MD, Sherr E, Muller D. Photochemical kinetics of corneal crosslinking with riboflavin. Invest Ophthalmol Vis Sci. 2012;53(4):2360-2367. doi:10.1167/iovs.11-9385 
26. Richoz O, Hammer A, Tabibian D, Gatzioufas Z, Hafezi F. The Biomechanical Effect of Corneal Collagen Cross-Linking (CXL) With Riboflavin and UV-A is Oxygen Dependent. Transl Vis Sci Technol. 2013;2(7):6. doi:10.1167/tvst.2.7.6

27. Pavel Kamaev, Mikhail Smirnov, Marc D Friedman, William A Eddington, Sarah Peterson, Evan A Sherr, David Muller; Photochemical kinetics model of corneal crosslinking with riboflavin. Invest. Ophthalmol. Vis. Sci. 2015;56(7):3004

28. Filippello M, Stagni E, O’Brart D. Transepithelial corneal collagen crosslinking: bilateral study. J Cataract Refract Surg. 2012;38(2):283-291. doi:10.1016/j.jcrs.2011.08.030

29. Kymionis GD, Tsoulnaras KI, Grentzelos MA, et al. Corneal stroma demarcation line after standard and high-intensity collagen crosslinking determined with anterior segment optical coherence tomography. J Cataract Refract Surg. 2014;40(5):736-740. doi:10.1016/j.jcrs.2013.10.029

30. Spoerl E, Mrochen M, Sliney D, Trokel S, Seiler T. Safety of UVA-riboflavin crosslinking of the cornea. Cornea. 2007;26(4):385-389. doi:10.1097/ICO.0b013e3180334f78 


\section{LEGENDS}

Figure 1: Oxygen delivery mask, placed on treated eye during PiXL procedure

Figure 2: UVA delivery device (Mosaic, Avedro Inc., Waltham, MS, USA) with pupil tracking system

Figure 3: Mean change in Manifest Refraction Spherical Equivalent (D) over the 6 postoperative months

Figure 4: Change in UDVA

A. Mean change in Uncorrected Distance Visual Acuity $(\log$ MAR) over the 6 postoperative months

B. Change in lines of UDVA at 6 months

C. Comparaison between postoperative and preoperative UDVA

Figure 5: Mean change in mean Keratometry (D) over the 6 postoperative months

Figure 6: Percentage of eyes with corneal flattening in TE-PiXL and PRK

Figure 7: Difference axial front curvature map at 6 months versus baseline

Figure 8: Anterior segment optic coherence topography at 1 month. Arrows show demarcation line

A. $6 \mathrm{~mm}$ whole thickness corneal hyperreflectivity

B. well delimited anterior stromal hyperreflectivity

Figure 9: Correlation of demarcation line depth at 1 month to

A. mean change in meam keratometry

B. mean change in Manifest Refraction Spherical Equivalent

Figure 10: Specular microscopy photography at 1 month showing endothelial cell count of 2652 cells $/ \mathrm{mm}^{2}$ although blurred image in TE-PiXL eye 
Figure 11: Slit lamp photography showing a circular stromal haze at 1 month. 


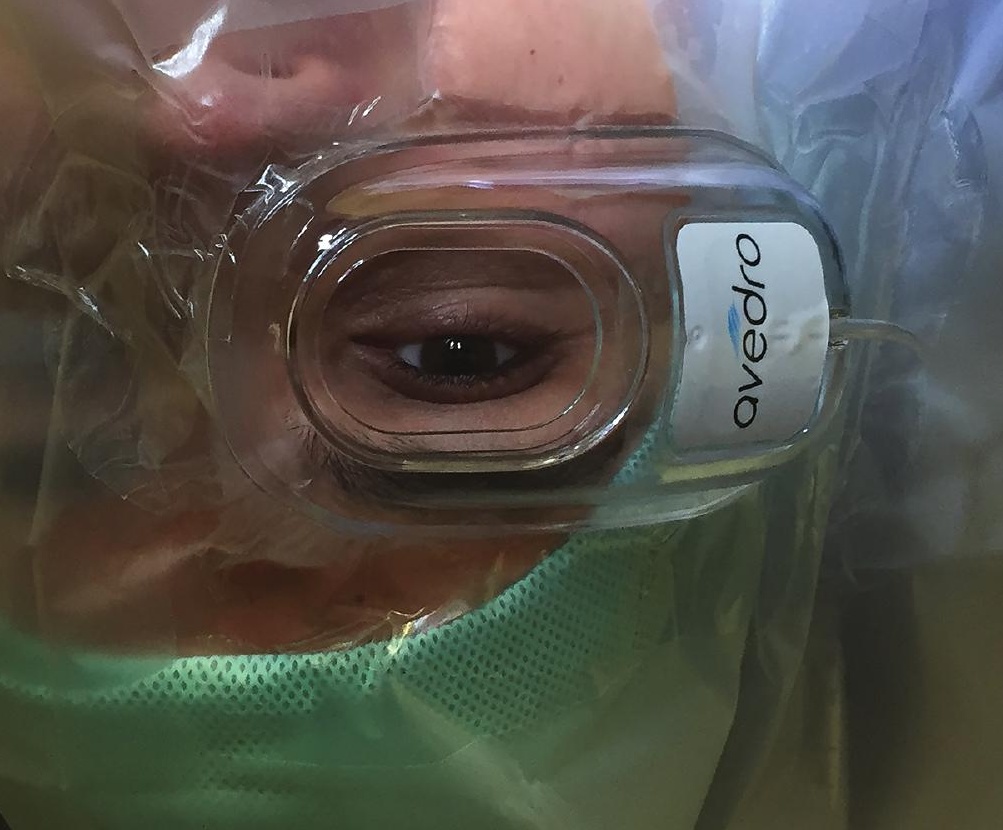




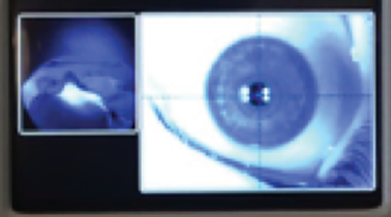




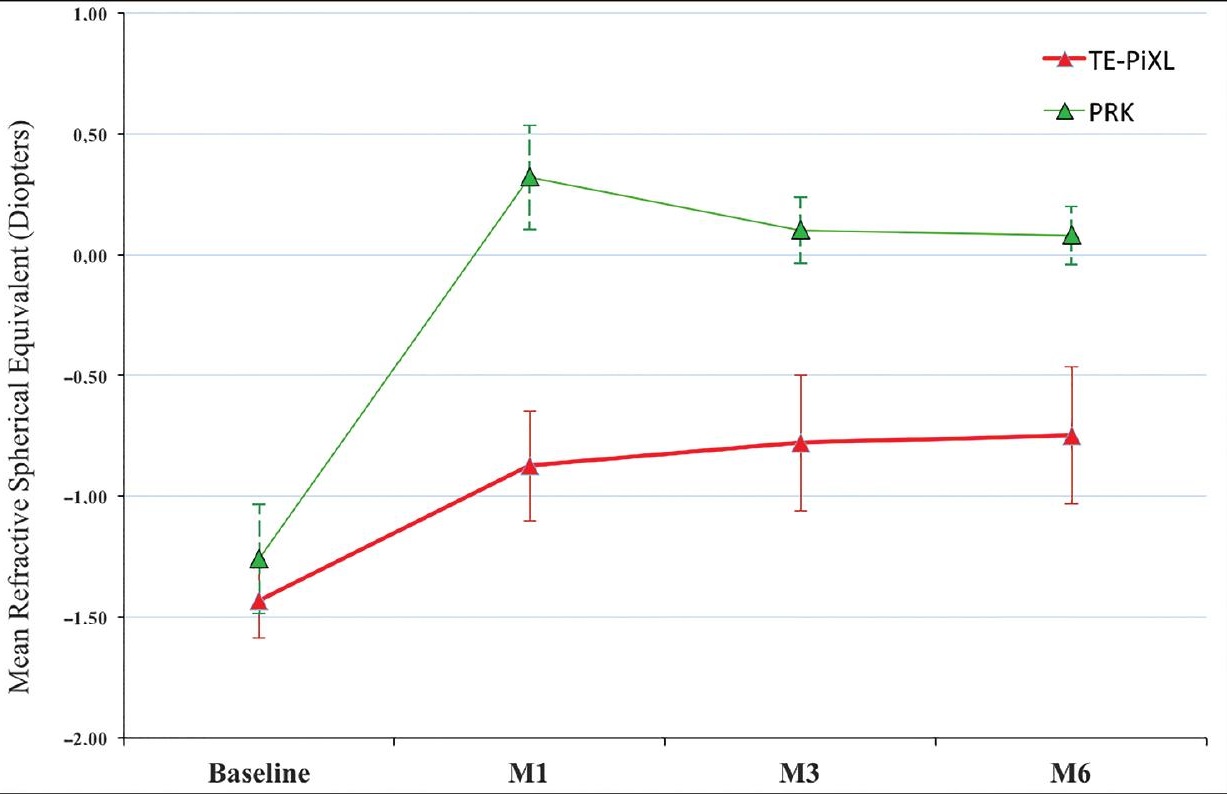




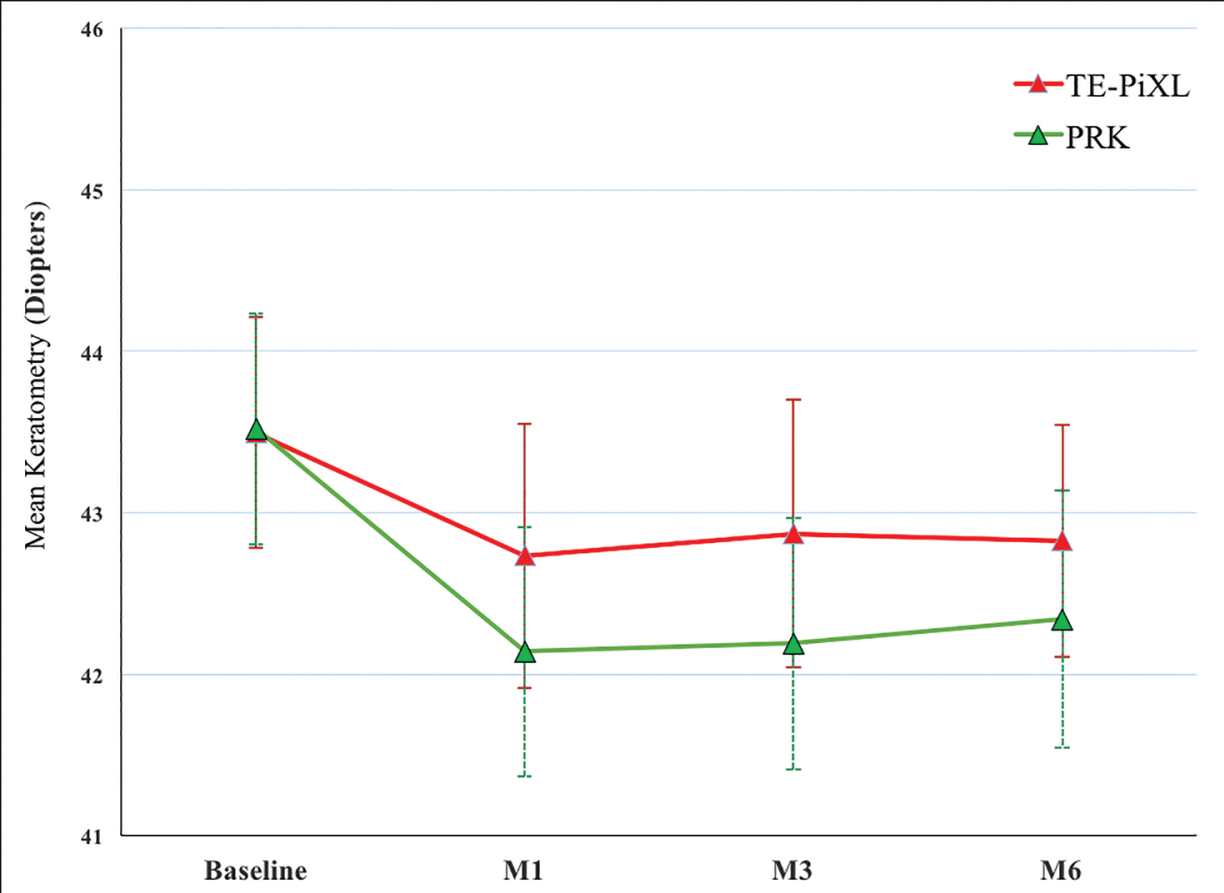




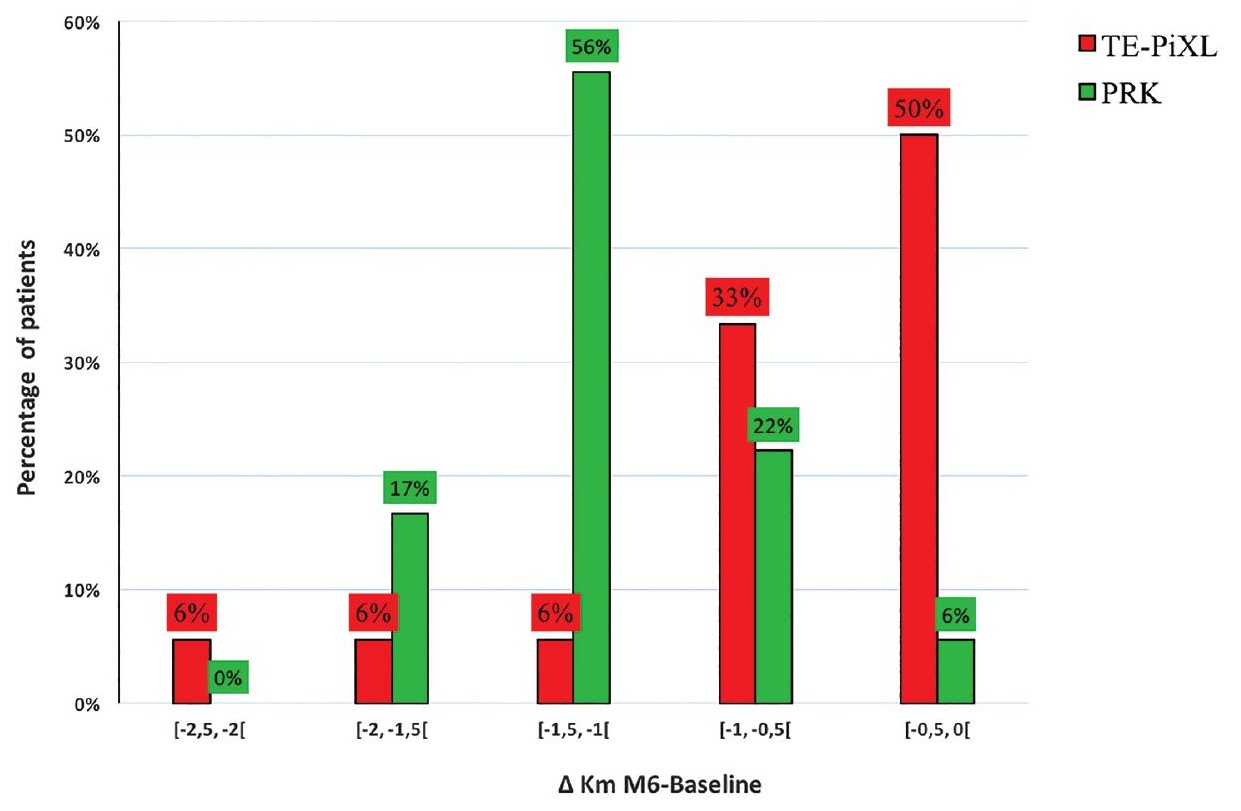




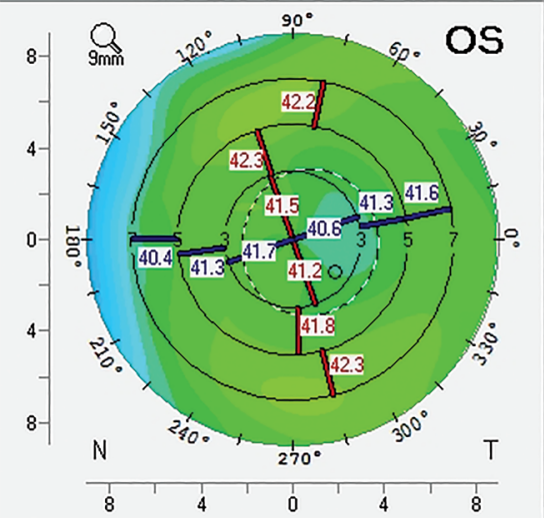

Carte Axiale/Sagittale (Face antérieure)

Carte Axiale/Sagillale (Face antérieure)

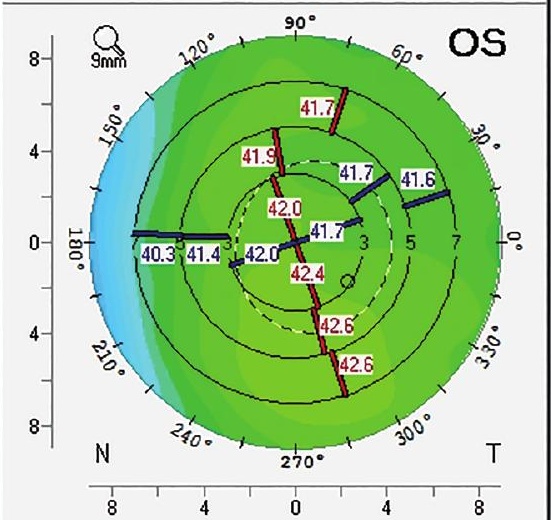

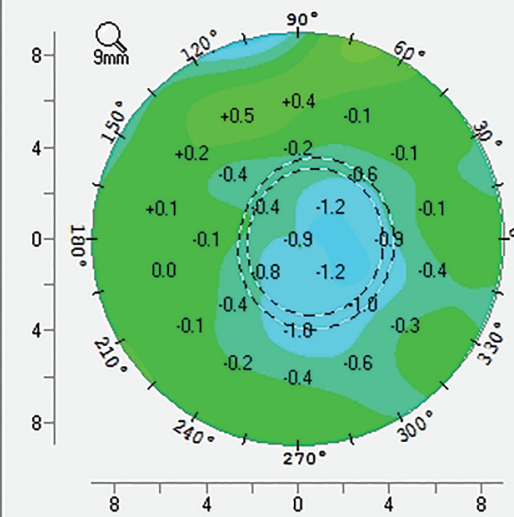




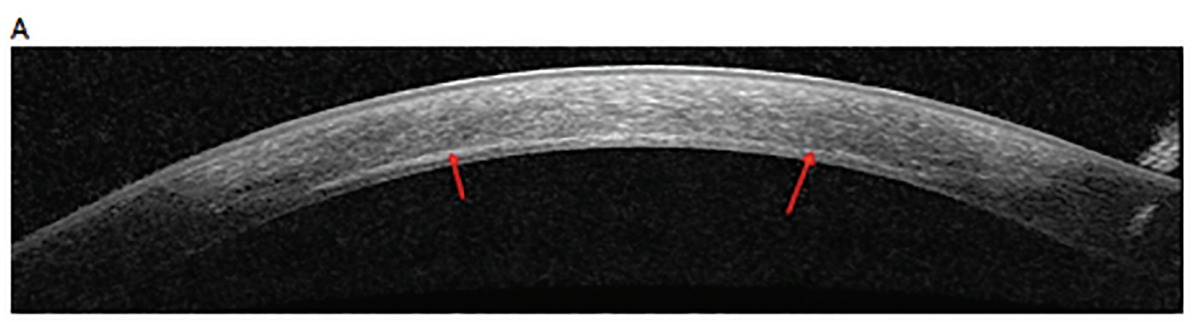

B

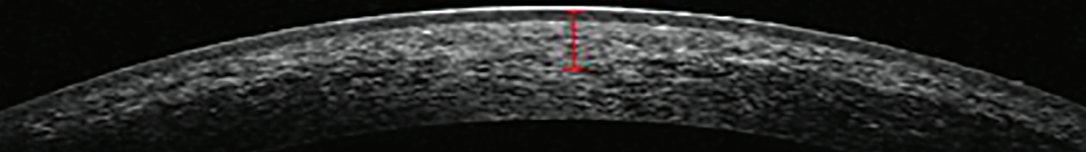



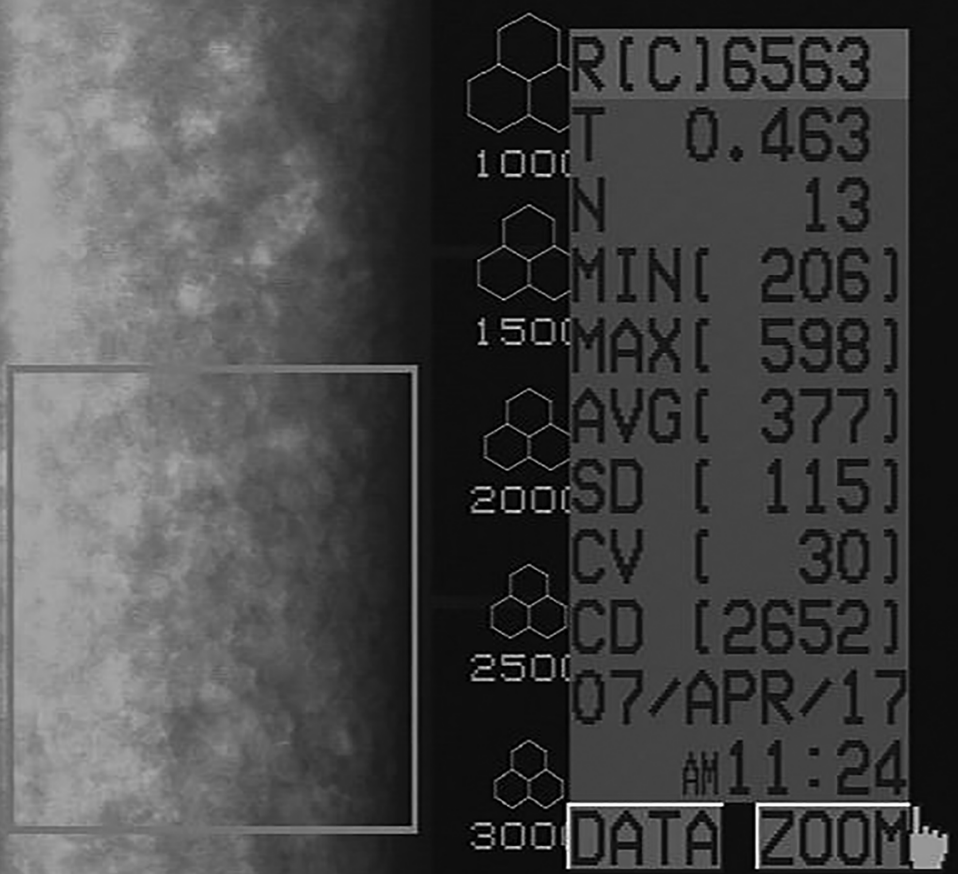


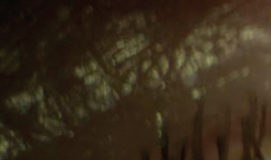
48 


\section{TABLES}

Table 1. Baseline clinical characteristics of TE-PiXL and PRK eyes.

\begin{tabular}{|c|c|c|c|c|}
\hline & & TE-PiXL & PRK & $\begin{array}{c}\mathrm{P} \\
\text { value }\end{array}$ \\
\hline \multicolumn{2}{|c|}{ MRSE (D) } & $\begin{array}{c}-1.43 \pm 0.31 \\
\text { [range: }-1 \text { to }-2 \text { ] }\end{array}$ & $\begin{array}{c}-1.26 \pm 0.35 \\
\text { [range: }-1 \text { to }-2 \text { ] }\end{array}$ & \multirow{11}{*}{$>0.05$} \\
\hline \multicolumn{2}{|c|}{ Spherical error (DS) } & $\begin{array}{c}-1.2 \pm 0.37 \\
\text { [range: }-0.5 \text { to }-2]\end{array}$ & $\begin{array}{c}-1.14 \pm 0.44 \\
{[\text { range: }-0.5 \text { to }-1.75 \text { ] }}\end{array}$ & \\
\hline \multicolumn{2}{|c|}{ Cylindrical error (DC) } & $\begin{array}{c}-0.42 \pm 0.25 \\
\text { [range: } 0 \text { to }-0.75]\end{array}$ & $\begin{array}{c}-0.4 \pm 0.26 \\
\text { [range: } 0 \text { to }-1]\end{array}$ & \\
\hline \multicolumn{2}{|l|}{$\mathrm{Km}(\mathrm{D})$} & $\begin{array}{c}43.49 \pm 1.43 \\
\text { [range: } 41 \text { to } 46.1]\end{array}$ & $\begin{array}{c}43.52 \pm 1.43 \\
\text { [range: } 41.3 \text { to } 46]\end{array}$ & \\
\hline \multicolumn{2}{|c|}{ Kmax (D) } & $\begin{array}{c}44.75 \pm 1.3 \\
\text { [range: } 42 \text { to } 46.8 \text { ] }\end{array}$ & $\begin{array}{c}44.5 \pm 1.36 \\
\text { [range: } 42.1 \text { to } 46.6 \text { ] }\end{array}$ & \\
\hline \multicolumn{2}{|c|}{ BCDVA (LogMAR) } & $\begin{array}{c}1.00 \pm 0.0 \\
\text { [range: } 1 \text { to } 1]\end{array}$ & $\begin{array}{c}1.00 \pm 0.0 \\
\text { [range: } 1 \text { to } 1]\end{array}$ & \\
\hline \multirow{2}{*}{ UDVA } & (LogMAR) & $\begin{array}{c}0.68 \pm 0.2 \\
\text { [range: } 0.2 \text { to } 1.3 \text { ] }\end{array}$ & $\begin{array}{c}0.67 \pm 0.19 \\
\text { [range: } 0.3 \text { to } 1.3 \text { ] }\end{array}$ & \\
\hline & Decimal & $\begin{array}{c}0.23 \pm 0.11 \\
\text { [range: } 0.05 \text { to } 0.63 \text { ] }\end{array}$ & $\begin{array}{c}0.23 \pm 0.08 \\
\text { [range: } 0.05 \text { to } 0.4 \text { ] }\end{array}$ & \\
\hline \multicolumn{2}{|c|}{ Pachymetry ( $\mu \mathrm{m})$} & $\begin{array}{c}537 \pm 28 \\
\text { [range: } 492 \text { to } 592 \text { ] }\end{array}$ & $\begin{array}{c}537 \pm 26 \\
\text { [range: } 489 \text { to } 576]\end{array}$ & \\
\hline \multicolumn{2}{|c|}{$\begin{array}{l}\text { Central epithelial thickness } \\
(\mu \mathrm{m})\end{array}$} & $\begin{array}{c}53 \pm 4 \\
\text { [range: } 49 \text { to } 56]\end{array}$ & $\begin{array}{c}53 \pm 4 \\
\text { [range: } 49 \text { to } 56]\end{array}$ & \\
\hline \multicolumn{2}{|c|}{$\begin{array}{l}\text { Endothelial cell count } \\
\left(\text { cells } / \mathrm{mm}^{2}\right)\end{array}$} & $\begin{array}{c}2654 \pm 371 \\
\text { [range: } 2030 \text { to } 3194]\end{array}$ & $\begin{array}{c}2692 \pm 395 \\
\text { [range: } 1788 \text { to } 3412 \text { ] }\end{array}$ & \\
\hline
\end{tabular}

MRSE = manifest refraction spherical equivalent $; \mathrm{D}=$ diopters $; \mathrm{DS}=$ diopter sphere $; \mathrm{DC}=$ diopter cylinder; $\mathrm{Km}=$ mean keratometry; $\mathrm{Kmax}=$ maximum simulated keratometry;

BCDVA = best corrected distance visual acuity; UDVA = uncorrected distance visual acuity Data are mean \pm standard deviation ; N.S.S = non-stastically significant

Table 2. Clinical characteristics of TE-PiXL and PRK eyes after 1, 3, and 6 months compared with baseline measurements.

\begin{tabular}{|c|c|c|c|c|c|c|c|}
\hline Parameter & Group & 1 month & $\mathrm{P} *$ value & 3 months & $\mathrm{P}^{*}$ value & 6 months & $\begin{array}{c}\mathrm{P}^{*} \\
\text { value }\end{array}$ \\
\hline \multirow{3}{*}{$\Delta \mathrm{MRSE}(\mathrm{D})$} & TE-PiXL & $0.55 \pm 0.30$ & $<0.001$ & $0.65 \pm 0.41$ & $<0.001$ & $0.72 \pm 0.42$ & $<0.001$ \\
\hline & PRK & $1.58 \pm 0.54$ & $<0.001$ & $1.37 \pm 0.42$ & $<0.001$ & $1.35 \pm 0.46$ & $<0.001$ \\
\hline & $\mathrm{P}^{\dagger}$ value & $<0.001$ & & $<0.001$ & & $<0.001$ & \\
\hline \multirow{3}{*}{$\begin{array}{l}\Delta \text { Sphere } \\
\text { (DS) }\end{array}$} & TE-PiXL & $0.47 \pm 0.32$ & $<0.001$ & $0.5 \pm 0.48$ & $<0.001$ & $0.57 \pm 0.47$ & $<0.001$ \\
\hline & PRK & $1.72 \pm 0.5$ & $<0.001$ & $1.4 \pm 0.43$ & $<0.001$ & $1.26 \pm 0.44$ & $<0.001$ \\
\hline & $\mathrm{P}^{\dagger}$ value & $<0.001$ & & $<0.001$ & & $<0.001$ & \\
\hline \multirow{3}{*}{$\begin{array}{l}\Delta \text { Cylinder } \\
\text { (DC) }\end{array}$} & TE-PiXL & $0.06 \pm 0.18$ & $>0.05$ & $0.07 \pm 0.12$ & $>0.05$ & $0.03 \pm 0.15$ & $>0.05$ \\
\hline & PRK & $-0.28 \pm 0.63$ & $>0.05$ & $0.03 \pm 0.57$ & $>0.05$ & $0.03 \pm 0.59$ & $>0.05$ \\
\hline & $\mathrm{P}^{\dagger}$ value & 0.03 & & $>0.05$ & & $>0.05$ & \\
\hline \multirow{2}{*}{$\begin{array}{l}\Delta \mathrm{UDVA} \\
(\mathrm{Log} M A R)\end{array}$} & TE-PiXL & $-0.34 \pm 0.17$ & $<0.001$ & $-0.35 \pm 0.19$ & $<0.001$ & $-0.35 \pm 0.21$ & $<0.001$ \\
\hline & PRK & $-0.65 \pm 0.19$ & $<0.001$ & $-0.67 \pm 0,2$ & $<0.001$ & $-0.66 \pm 0.19$ & $<0.001$ \\
\hline
\end{tabular}




\begin{tabular}{|c|c|c|c|c|c|c|c|}
\hline & $\mathrm{P}^{\dagger}$ value & $<0.001$ & & $<0.001$ & & $<0.001$ & \\
\hline \multirow{3}{*}{$\Delta \mathrm{Km}(\mathrm{D})$} & TE-PiXL & $-0.76 \pm 0.72$ & $<0.001$ & $-0.62 \pm 0.68$ & $<0.01$ & $-0.74 \pm 0.54$ & $<0.001$ \\
\hline & PRK & $-1.38 \pm 0.44$ & $<0.001$ & $-1.3 \pm 0.37$ & $<0.001$ & $-1.21 \pm 0.38$ & $<0.001$ \\
\hline & $\mathrm{P}^{\dagger}$ value & $<0.01$ & & $<0.001$ & & $<0.05$ & \\
\hline \multirow{3}{*}{$\begin{array}{l}\Delta \text { Endothelial } \\
\text { cell count } \\
\left(\text { cells } / \mathrm{mm}^{2}\right)\end{array}$} & TE-PiXL & $52 \pm 206.4$ & 0.424 & $-42 \pm 195$ & 0.402 & $-61 \pm 261$ & 0.355 \\
\hline & PRK & $42 \pm 276$ & 0.406 & $-32 \pm 208$ & 0.5 & $14.2 \pm 354$ & 0.786 \\
\hline & $\mathrm{P}^{\dagger}$ value & 0.952 & & 0.843 & & 0.445 & \\
\hline
\end{tabular}

MRSE $=$ manifest refraction spherical equivalent $; \mathrm{D}=$ diopters; $\mathrm{DS}=$ diopter sphere; $\mathrm{DC}=$ diopter cylinder; UDVA = uncorrected distance visual acuity; $\mathrm{Km}=$ mean keratometry; Data are mean \pm standard deviation.

*Comparing changes at 1,3 , and 6 months from baseline.

Comparing TE-PiXL and PRK eyes at the same time point.

Table 3. Depth of demarcation lines measures over time in TE-PiXL eyes

\begin{tabular}{|l|c|c|c|}
\hline & 1 month & 3 months & 6 months \\
\hline Central DLD \pm SD $(\mu \mathrm{m})$ & $366.1 \pm 133.3$ & $258.6 \pm 173.5$ & $220.5 \pm 199.2$ \\
\hline Mean peripheral DLD \pm SD $(\mu \mathrm{m})$ & $151.8 \pm 83$ & $113.9 \pm 89.7$ & $74.9 \pm 85.2$ \\
\hline
\end{tabular}

DLD $=$ Demarcation Line Depth 